clinical case of the rare ROHHAD syndrome which represents a condition related to central sleep apnea.

Objective To review the literature data on sleep-related breathing disorders and present our own clinical case of the rare ROHHAD syndrome.

Methods More than 100 literature sources were reviewed. Presented the case of a 6-year-old child with sleep apnea hospitalized to the Mordovian Republic Clinical Hospital.

Results ROHHAD syndrome is a very rare disease (about 100 cases are described worldwide). In most cases it presented as rapid obesity development since 3-5 years of age as well as hypoventilation, endocrine dysfunction, electrolyte disturbances. Autonomic nervous system dysfunction reveled in $\mathrm{ROH}-$ HAD pediatric patients rarely. Own observation demonstrated a typical clinical presentation of ROHHAD syndrome. Boy D., 6 years 11 months was admitted to the pediatric department of the Children's republican clinical hospital with severe weakness, fatigue during physical exertion, obesity, shortness of breath at rest, orthopnea, inability to sleep on the back (sleeps with an elevated head end or on the right side); lower extremities weakness during walking and climbing upstairs during the last year, changes in walking and in behavioral reactions. Cyanotic attacks and tachycardia have appeared during sleep in previous six months before admission. Firstly, the condition manifested with sudden and rapidly progressive obesity. Ventilation disorders became clinically evident after carried over pneumonia. Episodes of sleep apnea lasted up to 20 seconds, were accompanied by cyanotic attacks and tachycardia. Multiple investigations were performed to exclude systemic connective tissue diseases, neurological disturbances and brain pathology, muscular tissue pathology and metabolic diseases. Finally, the diagnosis was confirmed by the polysomnography and genetic tests. The ventilation support during sleep was prescribed.

Conclusion The rarity of the disease leads to a decreased awareness of pediatricians. The diagnosis is made by clinical picture and polysomnography, and timely diagnostics can increase the duration and improve the quality of life of patients.

\section{NEURORETINITIS DUE TO BARTONELLA HENSELAE INFECTION}

Monserrat Gutierrez*, Catalina Guerrero, Lucero Hagedorn, Valentina Kobus. Pontificia Universidad Católica de Chile

10.1136/archdischild-2021-europaediatrics.52

Introduction Bartonella henselae infection is a zoonosis with a worldwide distribution, cats are the natural reservoir and it predominates in children. In Chile, the prevalence of $\mathrm{B}$. henselae exposure is $13.3 \%$, determined by detection of specific IgG antibodies. The prevalence of serological screening in cats varies between 75 to $95 \%$, Cat scratch disease (CSD) is the most common form of presentation, characterized by regional lymphadenopathy that appears weeks after the scratch or bite of an infected cat. An atypical CSD may occur in some cases, and ocular involvement is a frequent manifestation, including Parinaud's syndrome and neuroretinitis.

Case Presentation A healthy 10-year-old woman, with a history of five days of fever, headache and fatigue, with spontaneous resolution. Days later, she developed persistent high-grade fever, asthenia, anorexia, neck pain with local inflammation and limp of the left leg, treated with Amoxicillin and antipyretics at home.

She was admitted to hospital with seven days of fever. Any localizing signs were found during physical examination. Erythrocyte sedimentation rate (ESR) and C-reactive protein levels were high. Chest radiography, trans-thoracic echocardiography, bone scintigraphy and left thigh ultrasound were normal; abdominal ultrasound was negative for hepatic abscesses. Blood culture, HIV test, immunological markers and QuantiFERON $^{\circledR}$-TB were negative; and $\operatorname{IgG}$ for B. Henselae revealed titers of 1: 1024, CSD was diagnosed. Searching for atypical CSD organ involvement, the left fundus exam found neuroretinitis with severe retinal compromise. The patient was finally treated with Azithromycin; and doxycycline with rifampin for 42 days.

Conclusion High suspicion should be given for the atypical presentation. Regarding the decision to treat optic neuropathy due to Bartonellosis, it is controversial, there are no randomized clinical studies that demonstrate visual improvement. Furthermore, it is a self-limiting condition in immunocompetent patients, which resolves in months.

\section{COVID-19 IN 18 MONTH OLD PATIENT}

Vedrana Zubčić Zrnc*, Zoran Barušić. General Hospital Ogulin, University Hospital for Infectious Diseases Dr. Fran Mihaljević

\subsection{6/archdischild-2021-europaediatrics.53}

Aim The aim of the following paper is to present the case of suspected COVID-19 in an 18 month old girl who manifested with typical signs of fever, cough, shortness of breath due to tachypnea and wheezing.

Methods As COVID-19 disease appeared very recently, the first reports in medical literature originate from PRC, showing that severe form is rare in pediatric patients. Most of the patients were asymptomatic (about 50\%) and the most commonly reported clinical finding was pneumonia.

Main Finding Our patient was tested at hospital admission. The first RT-PCR test on SARS-COV2, performed on oropharyngeal and nasopharyngeal swab, was positive. At that moment the child did not have typical laboratory findings for COVID-19. There was no evidence of leucopenia or lymphopenia, the CRP was mildly elevated and creatinine kinase $\mathrm{MB}$ was at the normal range. Radiological findings did not show bilateral ground-glass opacity. The child did not have a coexisting condition. The second PCR test taken on the fifth day of disease when all respiratory symptoms resolved was negative.

Conclusion In order to determine the spectrum of COVID 19 disease in young children we have to take into account possible co-infection with other respiratory viruses as well as the fact that some performed RT-PCR tests lead to false positive results.

\section{REFUSAL TO WALK: A CASE REPORT OF SPONDYLODISCITIS}

Sofia Branco*, Joana Lira, Beatriz Vieira, Maria José Dinis, Conceição Silva. Centro Hospitalar Póvoa de Varzim e Vila do Conde - Portugal

10.1136/archdischild-2021-europaediatrics.54 
Introduction Inflammation of the intervertebral disc and vertebral bodies is uncommon in children. The diagnosis can be challenging due to non-specific signs and symptoms.

Case Report A 12 month-old previously healthy female child presented with pain in the right hip, refusal to walk, and fever (up to $38.6^{\circ} \mathrm{C}$ ) for five days. There was no history of trauma. On admission, the infant was febrile and had a limping gait. The remainder of the physical examination was normal. She was hospitalized for further investigation. A complete blood count revealed

10880 white blood cells/ $\mu \mathrm{L}$ (52\% neutrophils, 34\% lymphocytes, $2 \%$ eosinophils and $10 \%$ monocytes), a haemoglobin level of $10,2 \mathrm{~g} / \mathrm{dL}$, and a platelet count of $452000 / \mu \mathrm{L}$. The erythrocyte sedimentation rate (ESR) was $73 \mathrm{~mm} /$ hour and the C-reactive protein was $2,74 \mathrm{mg} / \mathrm{dL}$. Radiography of the lower limbs and pelvis was normal. The following day, the child was unable to sit. Magnetic resonance imaging (MRI) of the spine showed an abnormal signal in the vertebral bodies and intervertebral disc at L4-L5, with a high signal on the STIR image, compatible with spondylodiscitis. Bed rest and empirical treatment with intravenous ceftriaxone and flucloxacillin was started.

Diagnostic tests performed to detect an infectious cause were negative, including blood and stool cultures, Wright's and Widal's tests, a Mantoux test, an Interferon-Gamma Release Assays blood test, a Polymerase Chain Reaction test for Mycobacterium tuberculosis from gastric washings, staining for acid-fast bacilli, and cultures for mycobacteria.

One week later, the levels of inflammatory markers decreased and the child improved clinically. An additional MRI study performed in the second week showed a reduction in signal alterations. After four weeks, the infant was siting and walking again without difficulty or pain. The inflammatory markers returned to normal. Intravenous therapy was switched to oral treatment with cefuroxime and flucloxacillin for another four weeks.

At a follow-up visit, an MRI study showed complete resolution of spondylodiscitis. There were residual abnormalities of the disc space and

L4/L5 vertebrae, with no functional significance. The child was asymptomatic and behaving normally.

Conclusion Suspicion of spondylodiscitis is important when a child presents with walking difficulties. The vascular nature of the intervertebral disc in children explains the predisposition to this rare condition. Magnetic resonance imaging of the spine is the gold standard for diagnosis. Early diagnosis is important to ensure a favourable outcome.

\section{REGRESSION OF MILESTONES IN AN INFANT AS PRESENTING FEATURE OF MATERNAL PERNICIOUS ANAEMIA}

Abhidhamma Kaninde*, M Katre, K Papadopoulou, R Ramaswamy. Leicester Royal Infirmary

\subsection{6/archdischild-2021-europaediatrics.55}

Background Children presenting with regression of milestones is not uncommon in the Paediatric population. One should be aware of wide range of differential diagnosis including neuro metabolic causes In our case, methylmalonic aciduria as a part was metabolic screening was noted leading to diagnosis of vitamin B12 deficiency in the infant and pernicious anaemia in mother.

Case Presentation We report the case of a 6-month-old female patient presenting with a history of suspected seizures in the form of abnormal upper limb movements, staring episodes with background history of developmental delay and regression of milestones over last few months.

There were no infection concerns. Birth history was normal and she was exclusively breastfed. She was well until four months of age when she presented with what sounded like shuddering episodes but had a normal examination that time.

On examination, she was hypo-reflexic and had poor grasp. Her tone was normal. She did not have any neurocutaneous markers. Our initial impression was of afebrile seizure likely infantile spasms.

Investigation Blood investigation revealed macrocytic anaemia. Her renal, liver and thyroid function were normal. Serum ammonia and lactate were normal.

An EEG and MRI brain were normal. Although we could not get Serum Amino acids results due to sampling error, her urine organic acid analysis revealed excessive excretion of Methyl malonic acid prompting further evaluation. Her B12 levels were noted to be significantly low, i.e. $<101 \mathrm{pg} / \mathrm{ml}$ (197-771) with normal folate levels With a history of exclusive breastfeeding, her mother was also investigated and found to have anti-parietal cell antibodies confirming underlying pernicious anaemia.

Patient had good recovery with normalisation of urine organic acid profile after treatment with injectable hydroxocobalmin.

Discussion Developmental delay is a common presentation in paediatric setting. Choo et al mentions prevalence up-to $15 \%$ amongst preschool children. One should take through history and undertake comprehensive examination for narrowing down wide spectrum of differential diagnosis. Global developmental delay, regression of milestone, presence of abnormal neurological examination are some of the red alarm signs which mandates further evaluation including neuroimaging and baseline metabolic work up.

In our case, methylmalonic aciduria lead us to diagnosis of nutritional vitamin B12 deficiency reemphasising importance of metabolic work up in these clinical presentations. Some studies like Hoznik et el mentions methylmalonic aciduria as most sensitive marker for Vitamin B12 deficiency.

Conclusion Vitamin B12 deficiency should be considered as an important differential while evaluating infants with developmental delay

\section{ACQUIRED TORTICOLLIS IN A TODDLER- A RARE DIAGNOSIS}

Abhidhamma Kaninde*, S Rasheed, M Aslam. Peterborough City Hospital

\subsection{6/archdischild-2021-europaediatrics.56}

Introduction Acquired torticollis although uncommon but is an important presentation in paediatric setting. One should be very thorough and sucpicious while evaluating these patients. If the torticolliis is non- traumatic it suggests more serious pathology. In our case, neck swelling with fever was pointing towards infective etiology but persistent symptoms made us 